\title{
Structure and Equivalence
}

\author{
Thomas William Barrett*
}

\begin{abstract}
It has been suggested that we can tell whether two theories are equivalent by comparing the structure that they ascribe to the world. If two theories posit different structures, then they must be inequivalent. The aim of this paper is to evaluate the extent to which this desideratum holds of the different standards of equivalence that are currently on the table.
\end{abstract}

\section{Introduction}

There is sometimes a sense in which two theories are equivalent. Equivalent theories say the same thing about the world, but might go about saying it in different ways. They are the same theory, just presented to us in different guises; they might, for example, use different mathematics or be formulated in different languages. The standard examples of equivalent theories are the Heisenberg and Schrödinger formulations of quantum mechanics and the Hamiltonian and Lagrangian formulations of classical mechanics. ${ }^{1}$

Since equivalent theories are supposed to be the same in all significant respects, we have a way to tell when two theories are inequivalent. If two theories differ in some significant respect, then they must be inequivalent. One way in which two theories might differ is in the structure that they ascribe to the world. For example, the Newtonian and Galilean theories of spacetime ascribe different amounts of structure to the world. Newtonian spacetime has the structure necessary to single out a rest frame, while Galilean spacetime does not come equipped with this structure. Since they ascribe different structure to spacetime, these two theories say different things about the world, and therefore must be inequivalent.

There are different standards of equivalence that are currently on the table, and the question of which one we should adopt is currently a subject of significant debate. The considerations about structure outlined above suggest a desideratum that we might use to evaluate standards of equivalence. In order for a particular standard of equivalence to be satisfactory, it should be the case

*Forthcoming in Philosophy of Science. I can be reached at tbarrett@philosophy.ucsb.edu. Thanks to Seb de Haro, Neil Dewar, Hans Halvorson, Laurenz Hudetz, and Jim Weatherall for helpful discussion.

${ }^{1}$ The latter case has recently been debated by North (2009), Curiel (2014), and Barrett (2015, 2017a). 
that if two theories are equivalent according to that standard, then they ascribe the same structure to the world.

Desideratum. If theories $T_{1}$ and $T_{2}$ are equivalent, then models of $T_{1}$ have the same structure as models of $T_{2}$.

A standard of equivalence fails to satisfy this desideratum just in case two theories can be equivalent according to that standard while nonetheless having models with different structure. Intuitively, if two theories ascribe different structure to the world, we can only infer that they are inequivalent if the standard of equivalence that we adopt satisfies this desideratum. Because we should be able to make this inference, the standard of equivalence that we adopt should satisfy the desideratum.

North (2009) has recently relied on the desideratum to argue, contrary to the standard view, that Hamiltonian and Lagrangian mechanics are inequivalent. If, as North (2009, p. 72) argues, there are "differences in structure" between the statespaces of two theories, then they must be inequivalent. Curiel (2014) agrees with North that Hamiltonian and Lagrangian mechanics are inequivalent. His argument is different than North's, but it also relies on something like the desideratum too. It turns on the idea that the two theories are inequivalent because "the underlying geometrical structures one uses to formulate each theory are not isomorphic" (Curiel, 2014, p. 1).

In what follows I will consider two standards of equivalence that are currently on the table and ask whether they satisfy the desideratum. In order to say whether they do, we need to make precise what it is for models of different theories to have the same structure. There are different ways to do this. My aim is to address these two issues - what standard of equivalence between theories we should adopt and what notion of sameness of structure between models we should adopt - in tandem, and work towards a reflective equilibrium between the two.

\section{The model isomorphism criterion}

We begin with a basic question: Under what conditions should we consider two theories equivalent? At the very least, equivalent theories should make the same empirical predictions. If we can distinguish between two theories on the basis of some observation, then we should not consider those theories equivalent.

The logical positivists are often credited with the view that empirical equivalence is not only necessary, but also sufficient for full equivalence of theories. Their idea was that the content of a theory is exhausted by its empirical content, so if two theories agree about the observable, this means that they have exactly the same content, which is just another way of saying that they are equivalent theories. It is now usually thought, however, that empirical equivalence is by itself too weak a standard of equivalence. In order to be equivalent, theories must share more in common than just their empirical predictions. 
The first standard that we will consider provides one way of making precise what else must be shared by two theories in order for them to be equivalent.

Criterion. Theories $T_{1}$ and $T_{2}$ are equivalent according to the model isomorphism criterion if every model of $T_{1}$ is isomorphic to a model of $T_{2}$, and vice versa.

The thought is that if two empirically equivalent theories are also equivalent according to the model isomorphism criterion, then this gives us good reason to think of them as saying the same thing about the world.

Two mathematical objects are said to be isomorphic if there is a bijection between them that preserves their basic structures. Isomorphism is the standard notion of 'sameness of structure' between mathematical objects. One therefore trivially sees that the model isomorphism criterion satisfies the desideratum. Indeed, there is a strong sense in which the desideratum leads one directly to this standard of equivalence. It is the standard that one arrives at when one uses the concept of isomorphism to make precise the relation 'same structure as' that appears in the desideratum.

It has recently been argued by Halvorson (2012) that adherents to the semantic view of theories are forced to endorse this standard of equivalence. And regardless of one's views on the debate between the semantic and syntactic views of theories, the idea behind the model isomorphism criterion is tempting. It is motivated by a desire to interpret a theory 'literally' or 'at face value.' If one interprets a theory this way, then one understands the mathematical structure of a theory's models as directly mirroring the structure of the world. Theories whose models are isomorphic are therefore 'saying the same thing' about the world. They are ascribing precisely the same structure to it. Conversely, theories whose models are not isomorphic must 'say different things' about the world.

Although the model isomorphism criterion satisfies the desideratum, it is a poor standard of equivalence. It judges too few pairs of theories to be equivalent. There are a number of examples of this, but for our purposes the following two will suffice. This first example was used by Winnie (1986) to demonstrate a similar point.

Example. The theory of linear orders can be formulated in two different ways. One formulation uses the concept of a non-strict order, while the other uses the concept of a strict order. In the first case, let $\Sigma_{1}=\{\leq\}$ be a signature where $\leq$ is a binary relation. The theory of linear orders $s_{1}$ is the $\Sigma_{1}$-theory with axioms saying that $\leq$ is transitive, antisymmetric, and that any two elements are comparable under the relation. In the second case, let $\Sigma_{2}=\{<\}$ be a signature where $<$ is a binary relation. The theory of linear orders 2 is the $\Sigma_{2}$-theory with axioms saying that $<$ is asymmetric, transitive, and trichotomous.

One can easily see that these two theories are not equivalent according to the model isomorphism criterion. Suppose that $M$ is a model of the theory of linear orders $_{1}$ and $N$ is a model of the theory of linear orders 2 . These two models are 
not isomorphic. ${ }^{2}$ There cannot be a bijection between $M$ and $N$ that preserves their basic structures because $\leq$ on $M$ is reflexive, while $<$ on $N$ is irreflexive (since it is asymmetric). Since models of these two theories are not isomorphic, they are not equivalent according to the model isomorphism criterion, despite the fact that they intuitively are the same. They both ascribe the same 'linear order structure' to sets, but they use different languages to do so.

The second example has been used to argue against the model isomorphism criterion before (Barrett, 2015, 2017a).

Example. It is well known that there are different ways to formulate general relativity. For example, it can be formulated on a smooth manifold with metric of signature (1-3), and it can be formulated on a smooth manifold with metric of signature (3-1). There is a strong sense in which these two formulations of general relativity are equivalent. Indeed, they only differ with respect to a choice of sign convention (i.e. a choice of whether to assign positive or negative length to timelike vectors).

These two formulations of general relativity are inequivalent according to the model isomorphism criterion. Manifolds with metric can only be isomorphic if their metrics have the same signature. Since models of these two formulations of general relativity employ metrics of different signatures, they are not isomorphic, and the two theories are therefore inequivalent according to the model isomorphism criterion.

These examples yield two conclusions. First, they show that the model isomorphism criterion is too strict a standard of equivalence. There are pairs of theories that are inequivalent according to the model isomorphism criterion which we nonetheless want to consider equivalent. In moving from our desideratum to the model isomorphism criterion, we have employed too strict a notion of 'same structure'. One wants to say that a model of the theory of linear orders 1 has the same structure as a model of the theory of linear orders 2 . Similarly, one wants to say that a model of (1-3) general relativity and a model of (3-1) general relativity have the same structure. These two examples simply do not strike one as cases in which the desideratum can be applied in order to conclude that the theories are inequivalent. But in both of the examples, the models of the theories in question are not isomorphic. This brings us to our second conclusion. When asking questions of equivalence - and when trying to clarify the desideratum - one should not make sameness of structure precise by using the concept of isomorphism. There are objects that are not isomorphic but which we nonetheless want to say have the same structure. Examples like the ones above demonstrate the "inadequacy of isomorphism as the criterion of structural equivalence" (Winnie, 1986, p. 128).

\footnotetext{
${ }^{2}$ In fact, the standard notion of isomorphism in model theory only applies to models that are in the same signature, so there is a sense in which $M$ and $N$ are trivially not isomorphic.
} 


\section{Categorical equivalence}

It is important to address both of these points. Before discussing how one might appropriately weaken the notion of isomorphism in order to capture a more adequate notion of 'sameness of structure', it will be useful to get a more liberal standard of theoretical equivalence on the table. There are many to choose from, but in the last few years one of the most discussed standards of equivalence has been categorical equivalence. This criterion traces back to Eilenberg and Mac Lane (1942, 1945), but was only recently introduced into philosophy of science by Halvorson $(2012,2016)$ and Weatherall (2016). It has since been applied to many cases of interest in physics. ${ }^{3}$

We need to do a bit of work to define categorical equivalence. First note that the class of models of a theory often has the structure of a category. We will call this the category of models of the theory. A category $C$ is a collection of objects with arrows between the objects that satisfy some basic properties. The arrows in a category $C$ can be thought of as the 'structure-preserving maps' between the objects of the category. An object in the category of models of a theory is just a model of that theory. The arrows between objects in the category of models encode the relationships that different models of the physical theory might bear to one another. If $T$ is a first-order theory formulated in a signature $\Sigma$, the category of models $\operatorname{Mod}(T)$ of $T$ has models of $T$ as its objects and elementary embeddings between models of $T$ as its arrows. ${ }^{4}$

A functor $F: C \rightarrow D$ is a structure-preserving map between categories $C$ and $D$. One can think of a functor as a 'translation' from objects and arrows of $C$ to objects and arrows of $D$. A functor $F: C \rightarrow D$ is full if for all objects $c_{1}, c_{2}$ in $C$ and arrows $g: F c_{1} \rightarrow F c_{2}$ in $D$ there exists an arrow $f: c_{1} \rightarrow c_{2}$ in $C$ such that $F f=g$. $F$ is faithful if $F f=F g$ implies that $f=g$ for all arrows $f: c_{1} \rightarrow c_{2}$ and $g: c_{1} \rightarrow c_{2}$ in $C$. $F$ is essentially surjective if for every object $d$ in $D$ there exists an object $c$ in $C$ such that $F c \cong d$. A functor $F: C \rightarrow D$ that is full, faithful, and essentially surjective is called an equivalence. The categories $C$ and $D$ are equivalent if there exists an equivalence between them. This gives us the following standard of equivalence between theories.

Criterion. Theories $T_{1}$ and $T_{2}$ are categorically equivalent if their categories of models $\operatorname{Mod}\left(T_{1}\right)$ and $\operatorname{Mod}\left(T_{2}\right)$ are equivalent.

When $T_{1}$ and $T_{2}$ are physical theories, one also requires that the functor realizing this equivalence 'preserves the empirical content of the theories' in some sense (Weatherall, 2016; Barrett, 2017a). This additional requirement is meant to guarantee that the theories in question are empirically equivalent, and moreover, that their empirical equivalence is respected by the functors that translate between the theories. Categorical equivalence is capturing a sense in which two theories are 'intertranslatable'; one can convert models of the one theory into models of the other theory, and these translations preserve many of the theoretical properties that one might take to be significant.

\footnotetext{
${ }^{3}$ For a review of recent work see Weatherall (2019) and the references therein.

${ }^{4}$ For further details see Barrett and Halvorson (2016b) and the references therein.
} 
In asking whether or not categorical equivalence satisfies our desideratum, we are asking whether the structure of the models of the theories is preserved under these translations. If we adopt isomorphism as our notion of sameness of structure, then categorical equivalence does not satisfy the desideratum. The two examples above show this. Models of the theory of orders ${ }_{1}$ are not isomorphic to models of the theory of orders 2 , but these two theories are nonetheless categorically equivalent. (It is well-known that these two theories are definitionally equivalent, and definitional equivalence entails categorical equivalence (Barrett and Halvorson, 2016b).) Similarly, models of 1-3 general relativity are not isomorphic to models of 3-1 general relativity, but these two theories are categorically equivalent too (Barrett, 2017a).

As we discussed above, however, these two examples give us good reason to refrain from adopting isomorphism as our notion of sameness of structure between models. One wants to say, for example, that a model of 1-3 general relativity and a model of 3-1 general relativity have the same structure, despite the fact that they are not isomorphic. This structure is simply displayed by the two models in two different ways, each corresponding to a choice of sign convention. We therefore want a more liberal notion of sameness of structure according to which these models have the same structure.

One such notion is suggested by the following considerations. A mathematical object comes equipped with more structure than just its 'basic level' of structure. It comes equipped with more structure than just that which we choose to display in its notation. In particular, it is natural to think of all of the structures that an object defines as 'coming for free' on the object. One example of this is the case of a metric space $(X, d)$. A metric space naturally comes equipped with - indeed, the metric defines - a canonical topology $\tau_{d}$. This topology $\tau_{d}$ is just as much a part of the structure of $(X, d)$ as the metric $d$ is.

Similarly, a model $\left(M, g_{a b}\right)$ of 1-3 general relativity comes equipped with additional structures other than just the 1-3 metric $g_{a b}$. One of these additional structures that its basic level of structure defines is the 3-1 metric $-g_{a b}$. This means that a model of 1-3 general relativity can define all of the structures that a model of 3-1 general relativity has, and vice versa. These models therefore have the same structure in the sense that each can define all of the structures of the other. The same holds of models of the two formulations of the theory of linear orders. The idea here is simple. Objects that are isomorphic have the same basic level of structure. But a mathematical object comes equipped with additional definable structures, and it might be - as in these two examples that once we take these into account we see that two non-isomorphic objects have precisely the same structure.

In order to make this notion of sameness of structure precise, we need to say what it means for two objects to define each others' structure. Suppose that $\Sigma_{1}$ and $\Sigma_{2}$ are signatures. (We assume without loss of generality that they only contain predicate symbols.) The elements of $\Sigma_{1}$ and $\Sigma_{2}$ encode the 'basic structures' on the two objects that we will consider. Let $A$ be a $\Sigma_{1}$-structure and $B$ a $\Sigma_{2}$-structure. If $q \in \Sigma_{2}$ is one of the structures on $B$, then we say 
that the $\Sigma_{1}$-structure $A$ explicitly defines $q^{B}$ if there is a $\Sigma_{1}$-formula $\phi$ such that $\phi^{A}=q^{B}$. Similarly, the $\Sigma_{2}$-structure $A$ explicitly defines $p^{A}$ if there is a $\Sigma_{2}$-formula $\psi$ such that $\psi^{B}=p^{A}$.

We can now state the following notion of sameness of structure. ${ }^{5}$

Definition. $A$ and $B$ are codeterminate if $A$ explicitly defines $q^{B}$ for every $q \in \Sigma_{2}$ and $B$ explicitly defines $p^{A}$ for every $p \in \Sigma_{1}$.

Codeterminate objects can define each others structures, and intuitively, they "differ only in their choice of exhibited relations" (Winnie, 1986, p. 75). For example, the model $A=(\mathbb{N}, \leq)$ of the theory of linear orders ${ }_{1}$ and the model $B=(\mathbb{N},<)$ of the theory of linear orders 2 , where $\leq$ and $<$ are given their usual extensions on the natural numbers, are codeterminate, despite the fact that they are not isomorphic. One can easily verify that $(x \leq y \wedge x \neq y)^{A}=(x<y)^{B}$, which means that $A$ explicitly defines all of the elements of $\Sigma_{2}$. One can also verify that $B$ explicitly defines $\leq$. The models $A$ and $B$ have the same structure. Indeed, they are both simply the natural numbers under their usual ordering. The only difference between them is in the way that they choose to display this structure. The former displays it by exhibiting the relation $\leq$, the latter by exhibiting the relation $<$, and each of these defines the other.

We can now ask whether categorically equivalent theories have codeterminate models. In other words, we want to know whether the desideratum holds of categorical equivalence when we take codetermination as our standard of sameness of structure. The aim of the remainder of this section is show that if we restrict our attention to a particular class of 'well behaved' equivalences between theories, then the answer to this question is yes.

We need some basic preliminaries in order to characterize these well behaved functors. A reconstrual $F$ of $\Sigma_{1}$ into $\Sigma_{2}$ is a map from the elements of the signature $\Sigma_{1}$ to $\Sigma_{2}$-formulas that takes an $n$-ary predicate symbol $p \in \Sigma_{1}$ to a $\Sigma_{2}$-formula $F p\left(x_{1}, \ldots, x_{n}\right)$ with $n$ free variables. ${ }^{6}$ A reconstrual $F: \Sigma_{1} \rightarrow$ $\Sigma_{2}$ extends to a map from arbitrary $\Sigma_{1}$-formulas to $\Sigma_{2}$-formulas in the usual recursive manner. If $T_{1}$ and $T_{2}$ are theories in the signatures $\Sigma_{1}$ and $\Sigma_{2}$, then we say that a reconstrual $F: \Sigma_{1} \rightarrow \Sigma_{2}$ is a translation $F: T_{1} \rightarrow T_{2}$ if $T_{1} \vDash \phi$ implies that $T_{2} \vDash F \phi$ for every $\Sigma_{1}$-sentence $\phi$. A translation $F$ gives rise to a map $F^{*}: \operatorname{Mod}\left(T_{2}\right) \rightarrow \operatorname{Mod}\left(T_{1}\right)$, which takes models of the theory $T_{2}$ to models of the theory $T_{1}$. One can show that $M$ and $F^{*}(M)$ are related to one another in the following way.

Lemma. Let $M$ be a model of $T_{2}$ and $\phi\left(x_{1}, \ldots, x_{n}\right)$ a $\Sigma_{1}$-formula. Then $M \vDash$ $F \phi\left[a_{1}, \ldots, a_{n}\right]$ if and only if $F^{*}(M) \vDash \phi\left[a_{1}, \ldots, a_{n}\right]$.

The map $F^{*}$ naturally extends to a mapping on elementary embeddings so that $F^{*}: \operatorname{Mod}\left(T_{2}\right) \rightarrow \operatorname{Mod}\left(T_{1}\right)$ is a functor between the categories of models

\footnotetext{
${ }^{5}$ This notion of codetermination was proposed by Winnie (1986), though he uses a variety of implicit definability instead of explicit definability. For more details on the relation between the two kinds of definability, see Winnie (1986) or Barrett (2017b).

${ }^{6}$ See Barrett and Halvorson (2016a) for a more comprehensive treatment of reconstruals and translations.
} 
of $T_{2}$ and $T_{1}$. If $f: M \rightarrow N$ is an arrow between models of $T_{2}$, then we define $F^{*}(f)=f$. One uses the Lemma to verify that $F^{*}(f)$ is an elementary embedding. This means that a translation $F: T_{1} \rightarrow T_{2}$ gives rise to a functor $F^{*}: \operatorname{Mod}\left(T_{2}\right) \rightarrow \operatorname{Mod}\left(T_{1}\right)$. Functors $F^{*}$ that arise from translations in this manner will be our 'well behaved' functors that we consider.

We can now state the following result.

Proposition. Let $\Sigma_{1}$ and $\Sigma_{2}$ be disjoint signatures. If $F: T_{2} \rightarrow T_{1}$ is a translation from the $\Sigma_{2}$-theory $T_{2}$ to the $\Sigma_{1}$-theory $T_{1}$ such that $F^{*}: \operatorname{Mod}\left(T_{1}\right) \rightarrow$ $\operatorname{Mod}\left(T_{2}\right)$ is a categorical equivalence, then $M$ and $F^{*}(M)$ are codeterminate for every model $M$ of $T_{1}$.

We need a few more definitions before proving this proposition. If $\Sigma \subset \Sigma^{+}$ are signatures, we say that a $\Sigma^{+}$-theory $T^{+}$is an extension of a $\Sigma$-theory $T$ if $T \vDash \phi$ implies that $T^{+} \vDash \phi$ for every $\Sigma$-sentence $\phi$. When $T^{+}$is an extension of a $\Sigma$-theory $T$, we can define the projection functor $\Pi: \operatorname{Mod}\left(T^{+}\right) \rightarrow \operatorname{Mod}(T)$ by

$$
\Pi(M)=\left.M\right|_{\Sigma} \quad \Pi(h)=h
$$

for every model $M$ of $T^{+}$and elementary embedding $h$ between models of $T^{+}$. Here $\left.M\right|_{\Sigma}$ is the $\Sigma$-structure obtained from $M$ by forgetting the extensions of all the predicates not in $\Sigma$.

Proof. Let $M$ be a model of $T_{1}$. If $q \in \Sigma_{2}$ is a predicate symbol, then the Lemma implies that $q^{F^{*}(M)}=F q^{M}$, which means that $M$ explicitly defines $q$.

It takes more work to show that $F^{*}(M)$ defines all of the structures of $M$. Consider the $\Sigma_{1} \cup \Sigma_{2}$-theory $T_{1}^{+}$that is defined as follows:

$$
T_{1} \cup\left\{\forall x(q(x) \leftrightarrow F q(x)): q \in \Sigma_{2}\right\}
$$

$T_{1}^{+}$is a definitional extension of $T_{1}$. Using the fact that $F$ is a translation, one can show that $T_{1}^{+}$is an extension of $T_{2}$. One can then verify using the Lemma that the following diagram commutes, where $\Pi_{1}: \operatorname{Mod}\left(T_{1}^{+}\right) \rightarrow \operatorname{Mod}\left(T_{1}\right)$ and $\Pi_{2}: \operatorname{Mod}\left(T_{1}^{+}\right) \rightarrow \operatorname{Mod}\left(T_{2}\right)$ are the projection functors.

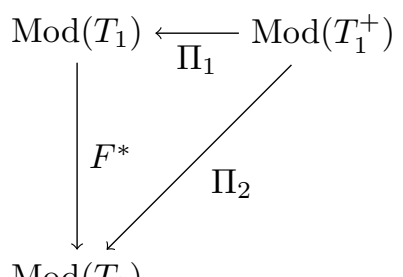

$\operatorname{Mod}\left(T_{2}\right)$

Since $T_{1}^{+}$is a definitional extension of $T_{1}, \Pi_{1}$ is an equivalence (Barrett and Halvorson, 2016b, Propositions 5.1-3). By assumption $F^{*}$ is an equivalence, so this means that $\Pi_{2}$ must be an equivalence too.

Now using the fact that $\Pi_{2}$ is an equivalence, Beth's theorem - in particular a simple corollary to it (Barrett, 2017b, Corollary 1) — implies that for every 
predicate symbol $p \in \Sigma_{1}$ there is a $\Sigma_{2}$-formula $\psi$ such that $T_{1}^{+} \vDash \forall x(p(x) \leftrightarrow$ $\psi(x))$. This gives us the following string of equivalences:

$$
a \in p^{M} \Longleftrightarrow a \in p^{M^{+}} \Longleftrightarrow a \in \psi^{M^{+}} \Longleftrightarrow a \in \psi^{\Pi_{2}\left(M^{+}\right)} \Longleftrightarrow a \in \psi^{F^{*}(M)}
$$

(Here $M^{+}$is the unique model of $T_{1}^{+}$that satisfies $\Pi_{1}\left(M^{+}\right)=M$.) The first equivalence follows from the definition of $\Pi_{1}$, the second from our choice of $\psi$, the third the definition of $\Pi_{2}$, and the fourth from the fact that the above diagram commutes. This means that $p^{M}=\psi^{F^{*}(M)}$, so $F^{*}(M)$ defines all of the structures of $M$, and the two models are therefore codeterminate.

This proposition shows us that if two theories are categorically equivalent and the functor realizing the equivalence is suitably well behaved - in the sense that it arises from a translation between the theories - then their models are codeterminate. $^{7}$ This demonstrates a sense in which categorical equivalence satisfies our desideratum. Insofar as we take codetermination as our standard of sameness of structure, this result gives us reason to say that categorically equivalent theories have models with the same structure.

\section{What is it to interpret a theory literally?}

My aim in this last section is to address a worry that one might have about categorical equivalence and other liberal standards of equivalence. The worry is that if one endorses a standard of equivalence that is so liberal, then one is forced away from scientific realism.

In order to be a scientific realist, one needs to take our best scientific theories 'literally' or 'at face value' (van Fraassen, 1980, p. 8). The most popular example of a non-literal interpretation of our scientific theories traces back to the logical positivists, whose view van Fraassen (1980, p. 10-11) describes as follows.

On the positivists' interpretation of science, theoretical terms have meaning only through their connection with the observable. Hence they hold that two theories may in fact say the same thing although in form they contradict each other. (Perhaps the one says that all matter consists of atoms, while the other postulates instead a universal continuous medium; they will say the same thing nevertheless if they agree in their observable consequences, according to the positivists.) But two theories which contradict each other in such a way can 'really' be saying the same thing only if they are not literally construed.

The positivists' non-literal interpretation of theories goes hand-in-hand with their commitment to empirical equivalence as the proper standard of equivalence between theories. One might therefore worry that proponents of liberal

\footnotetext{
${ }^{7}$ Hudetz (2017) contains similar results which point to exactly the line of inquiry that leads one to the above proposition. The standard of equivalence that he proposes, called definable categorical equivalence, builds in 'by hand' the requirement that models be mapped to codeterminate models.
} 
standards of equivalence like categorical equivalence are in a similar position as the positivists. If there are categorically equivalent theories that contradict one another, then this would mean that committing to categorical equivalence forces us to take our theories non-literally, which in turn forces us away from realism.

At first glance, one might worry that there are categorically equivalent theories, like the following pair, that contradict one another when taken literally.

Example. General relativity is normally formulated geometrically by using a smooth manifold with various structures on it. But in the early 1970s, Geroch (1972) noticed that general relativity could also be formulated in a purely algebraic fashion by using something called an "Einstein algebra." One can think of the elements of an Einstein algebra as the smooth scalar functions on a spacetime.

Rosenstock et al. (2015) have recently shown that these two theories are categorically equivalent. But in this case one might be tempted to say that these two theories contradict one another if we take them literally. General relativity ascribes a kind of 'geometric structure' to the world, whereas the theory of Einstein algebras ascribes a kind of 'algebraic structure' to the world, and these are radically different kinds of structures. For example, when we draw models of these two theories (Barrett, 2017a), they look completely different, suggesting that the two theories contradict one another. Their models provide us with different pictures of the world.

There are other categorically equivalent theories that one might worry contradict one another, but this example will suffice for our purposes here. Simply put, the worry is the following. Categorically equivalent theories might contradict one another in terms of the structure they ascribe to the world, and if so, then adopting categorical equivalence as our standard of equivalence forces us into a form of anti-realism.

I would like to suggest that this worry about categorical equivalence and other liberal standards of equivalence is misplaced. The claim that, for example, general relativity and the theory of Einstein algebras contradict one another is subtly rooted in the idea that we should use isomorphism as our standard for when two objects have the same structure. If one takes isomorphism as the proper standard of sameness of structure between models, then one does indeed have good reason to think that categorically equivalent theories do literally contradict one another. An Einstein algebra and a smooth manifold with metric are not isomorphic, so the theories contradict one another in virtue of ascribing different structure to the world. But we have seen that isomorphism is not a satisfactory standard of sameness of structure.

If we adopt a reasonable notion of sameness of structure, then the idea that categorically equivalent theories contradict one another disappears. General relativity and the theory of Einstein algebras have codeterminate models. From the starting point of a smooth manifold with Lorentzian metric, one can build an Einstein algebra, and conversely, from the starting point of an Einstein algebra, one can build a smooth manifold with Lorentzian metric. There is therefore 
a strong sense in which these two theories do not contradict one another in terms of the structure they posit, so long as we adopt an appropriate notion of sameness of structure. If one claims that general relativity and the theory of Einstein algebras are inequivalent in virtue of the former positing geometric structure and the latter positing algebraic structure, then one is taking these theories too literally.

The proposition in the previous section guarantees that categorically equivalent theories — so long as the functor realizing the equivalence is suitably well behaved - will have have codeterminate models. So once we are clear about all of the structures that models have - in particular, by being clear about what structures are definable on these models - then we see that the models of categorically equivalent theories have precisely the same structure, despite the fact that they are not isomorphic. This means that adopting a more liberal standard of equivalence, like categorical equivalence, need not be understood as a move away from literal interpretation of our scientific theories. Rather, it seems that standards of equivalence that are much stricter than categorical equivalence are committing us to a kind of super-literal interpretation.

\section{References}

Barrett, T. W. (2015). On the structure of classical mechanics. The British Journal for the Philosophy of Science 66(4), 801-828.

Barrett, T. W. (2017a). Equivalent and inequivalent formulations of classical mechanics. Forthcoming in the British Journal for the Philosophy of Science.

Barrett, T. W. (2017b). What do symmetries tell us about structure? Manuscript.

Barrett, T. W. and H. Halvorson (2016a). Glymour and Quine on theoretical equivalence. Journal of Philosophical Logic 45(5), 467-483.

Barrett, T. W. and H. Halvorson (2016b). Morita equivalence. The Review of Symbolic Logic 9(3), 556-582.

Curiel, E. (2014). Classical mechanics is Lagrangian; it is not Hamiltonian. The British Journal for the Philosophy of Science 65(2), 269-321.

Eilenberg, S. and S. Mac Lane (1942). Group extensions and homology. Annals of Mathematics.

Eilenberg, S. and S. Mac Lane (1945). General theory of natural equivalences. Transactions of the American Mathematical Society.

Geroch, R. (1972). Einstein algebras. Comm. Math. Phys..

Halvorson, H. (2012). What scientific theories could not be. Philosophy of Science $79(2), 183-206$. 
Halvorson, H. (2016). Scientific theories. In P. Humphreys (Ed.), The Oxford Handbook of Philosophy of Science, pp. 585-608. Oxford University Press.

Hudetz, L. (2017). Definable categorical equivalence: Towards an adequate criterion of theoretical intertranslatability. Forthcoming in Philosophy of Science.

North, J. (2009). The 'structure' of physics: A case study. The Journal of Philosophy 106, 57-88.

Rosenstock, S., T. W. Barrett, and J. O. Weatherall (2015). On Einstein algebras and relativistic spacetimes. Studies in History and Philosophy of Science Part B: Studies in History and Philosophy of Modern Physics 52, 309-316.

van Fraassen, B. C. (1980). The Scientific Image. Oxford.

Weatherall, J. O. (2016). Are Newtonian gravitation and geometrized Newtonian gravitation theoretically equivalent? Erkenntnis 81(5), 1073-1091.

Weatherall, J. O. (2019). Theoretical equivalence in physics. Forthcoming in Philosophy Compass.

Winnie, J. (1986). Invariants and objectivity: A theory with applications to relativity and geometry. In R. G. Colodny (Ed.), From Quarks to Quasars, pp. 71-180. Pittsburgh: Pittsburgh University Press. 